\title{
Wigner Crystallization of a two dimensional electron gas in a magnetic field: single electrons versus electron pairs at the lattice sites
}

\author{
M. Taut \\ Institute for Solid State and Materials Research Dresden \\ POB 270016, 01171 Dresden, Germany \\ email: m.taut@ifw-dresden.de
}

\begin{abstract}
The ground state energy and the lowest excitations of a two dimensional Wigner crystal in a perpendicular magnetic field with one and two electrons per cell is investigated. In case of two electrons per lattice site, the interaction of the electrons within each cell is taken into account exactly (including exchange and correlation effects), and the interaction between the cells is in second order (dipole) van der Waals approximation. No further approximations are made, in particular Landau level mixing and incomplete spin polarization are accounted for. Therefore, our calculation comprises a, roughly speaking, complementary description of the bubble phase (in the special case of one and two electrons per bubble), which was proposed by Koulakov, Fogler and Shklovskii on the basis of a Hartree Fock calculation. The phase diagram shows that in GaAs the paired phase is energetically more favorable than the single electron phase for, roughly speaking, filling factor $f$ larger than 0.3 and density parameter $r_{s}$ smaller than 19 effective Bohr radii (for a more precise statement see Fig.s 4 and 5). If we start within the paired phase and increase magnetic field or decrease density, the pairs first undergo some singlet- triplet transitions before they break.
\end{abstract}

PACS: 71.10.Hf Non Fermi liquid ground states, 71.10.Li Excited states and pairing interactions in model systems, 73.40.Hm Fractional Quantum Hall Effect

\section{INTRODUCTION}

The Wigner crystal (WC) and the Fermi liquid are states of matter which are established already for decades. Later on, the Laughlin liquid as a special state in high magnetic fields has been added. In both liquid states, wave-functions of the electrons have an appreciable overlap. In the extreme Wigner crystal limit this overlap can be neglected. The issue of this paper is if there is a state between crystal and liquid, where a finite number of particles overlap as in the liquid, but interacts with the neighboring cluster as in the Wigner crystal (paired or clustered Wigner crystal). The charge density yzave (CDW) state, mostly investigated in Hartree Fock (HF) approximation, is a alternative intermediate phase. 1 - In the latter case, there is a density modulation, but the density is so high that overlap plays an important role. (Observe that in some papers the CDW state is also called WC.) What we call here 'paired Wigner crystal' can be viewed as a derivative of three other states. It is as (i) a Wigner crystall with two electronsper cell, (ii) a crystallized paired electron liquid known from superconductivity and theories for the $f=\frac{5}{2}$ state $\mathrm{g}^{2}$, (iii) a state of liquid islands, if the clusters are large.

The first paper which considered the possibility of a paired WC in 3 dimensions (3D) without a magnetic field is Ref. 10 using a cell approximation for the inter-cell interaction and a variational ansatz for the intra-cell problem. In a previous paper 11 it was shown, that the model of Ref. 10 can be solved exactly, even if fluctuation corrections in second order van der Waals approximation are included additionally. It turned out that in 3D without a magnetic field, the paired WC is energetically lower than the conventional WC for $r_{s}<9$, but in this density region the Fermi liquid is probably already lower than both WCs. More recently, this issue has been investigated in 2D with a magnetic field using the HF approximation 12 The result is that in higher Landau levels (LL) the ground states are phases with circular or stripy clusters. Meanwhile, numerical solutions for finite size systems have been published 13. 4 , which confirm these findings qualitatively within their model. All previous work on this issue neglect LL mixing and incomplete spin polarization. In the present approach, we investigated clusters with one and two electrons. In the latter case, the interaction within each cluster is exactly accounted for (including exchange and correlations). In both cases, the inter-cell interaction is considered in second order van der Waals approximation (no exchange, Coulomb interaction in dipole approximation). In Sect. II we describe the solution of the Schrödinger equation in more detail and in Sect. III our results are presented. Sect. IV gives a summary and an overview. 


\section{SOLUTION OF THE SCHRÖDINGER EQUATION}

The Hamiltonian of a system of $N$ electrons at each lattice site and a positive compensating background of density $n_{0}$ reads (atomic units $\hbar=m=e=1$ are adopted)

$$
H=T+V_{\text {intra }}^{e e}+V_{i n t e r}^{e e}+V^{e b}+V^{b b}
$$

with the kinetic energy

$$
T=\sum_{n} \sum_{k} \frac{1}{2 m^{*}}\left[\mathbf{p}_{n k}+\frac{1}{c} \mathbf{A}\left(\mathbf{u}_{n k}\right)\right]^{2}
$$

the intra-cell $\mathrm{e}-\mathrm{e}-$-interaction

$$
V_{\text {intra }}^{e e}=\sum_{n} \frac{1}{2} \sum_{k \neq k^{\prime}} \frac{\beta}{\left|\mathbf{u}_{n k}-\mathbf{u}_{n k^{\prime}}\right|}
$$

the inter-cell $\mathrm{e}-\mathrm{e}-$-interaction

$$
V_{\text {inter }}^{e e}=\frac{1}{2} \sum_{n \neq n^{\prime}} \sum_{k k^{\prime}} \frac{\beta}{\left|\left(\mathbf{R}_{n}^{0}-\mathbf{R}_{n^{\prime}}^{0}\right)+\left(\mathbf{u}_{n k}-\mathbf{u}_{n^{\prime} k^{\prime}}\right)\right|}
$$

the electron- background interaction

$$
V^{e b}=-\sum_{n} \sum_{k} \int d \mathbf{r}^{\prime} \frac{n_{0}}{\left|\mathbf{r}_{n k}-\mathbf{r}^{\prime}\right|}
$$

and the background- background interaction

$$
V^{b b}=\frac{1}{2} \int d \mathbf{r} \int d \mathbf{r}^{\prime} \frac{n_{0} n_{0}}{\left|\mathbf{r}-\mathbf{r}^{\prime}\right|}
$$

In these definitions, $m^{*}$ is the effective mass, $\beta$ the inverse background dielectric constant, $\mathbf{R}_{n}^{0}$ are the lattice sites, and $\mathbf{r}_{n k}=\mathbf{R}_{n}^{0}+\mathbf{u}_{n k}$ the electron coordinates. We use the symmetric gauge $\mathbf{A}=\frac{1}{2} \mathbf{B} \times \mathbf{u}$. Latin letters $n$ and $n^{\prime}$ denote the lattice sites and $k$ and $k^{\prime}$ the electrons within a cell (or cluster). If the distance between the cell centers is large compared with the average distance between the electrons within a cell, the inter-cell interaction can be expanded in a multi-pole series up to second order

$$
V_{\text {inter }}^{e e}=V_{\text {inter }}^{e e(0)}+V_{\text {inter }}^{e e(2)}
$$

with the zero and second order terms

$$
\begin{aligned}
& V_{\text {inter }}^{e e(0)}=N^{2} \frac{1}{2} \sum_{n \neq n^{\prime}} \frac{\beta}{\left|\mathbf{R}_{n}^{0}-\mathbf{R}_{n^{\prime}}^{0}\right|} \\
& V_{\text {inter }}^{e e(2)}=\frac{1}{N} \frac{1}{2} \sum_{n n^{\prime}} \sum_{k k^{\prime}} \mathbf{u}_{n k} \cdot \mathbf{C}_{n k, n^{\prime} k^{\prime}} \cdot \mathbf{u}_{n^{\prime} k^{\prime}}
\end{aligned}
$$

the force constant matrix

$$
\begin{aligned}
\mathbf{C}_{n k, n^{\prime} k^{\prime}} & =+\beta N^{2} \mathbf{T}_{0} \quad \text { for } \quad(n k)=\left(n^{\prime} k^{\prime}\right) \\
& =-\beta N \mathbf{T}_{n n^{\prime}} \quad \text { else }
\end{aligned}
$$

the dipole tensor

$$
\mathbf{T}_{n n^{\prime}}=\frac{1}{R_{n n^{\prime}}^{5}}\left[3 \mathbf{R}_{n n^{\prime}} \circ \mathbf{R}_{n n^{\prime}}-R_{n n^{\prime}}^{2} \mathbf{I}\right]
$$

and the definitions $\mathbf{T}_{0}=\sum_{n \neq 0} \mathbf{T}_{0 n}$ and $R_{n n^{\prime}}=\mathbf{R}_{n}^{0}-\mathbf{R}_{n^{\prime}}^{0}$. By rearrangement of terms we obtain the following form 


$$
H=E_{N}^{M a d}+\left(T+V_{i n t r a}^{e e}\right)+V_{\text {inter }}^{e e(2)}
$$

with the Madelung energy for a lattice with $N$ electrons at each lattice site

$$
E_{N}^{M a d}=N^{2} \frac{1}{2} \sum_{n \neq n^{\prime}} \frac{\beta}{\left|\mathbf{R}_{n}^{0}-\mathbf{R}_{n^{\prime}}^{0}\right|}-N \sum_{n} \int d \mathbf{r}^{\prime} \frac{n_{0}}{\left|\mathbf{R}_{n}^{0}-\mathbf{r}^{\prime}\right|}+\frac{1}{2} \int d \mathbf{r} \int d \mathbf{r}^{\prime} \frac{n_{0} n_{0}}{\left|\mathbf{r}-\mathbf{r}^{\prime}\right|}
$$

By introducing the center of mass (c.m.) coordinate $\mathbf{U}_{n}$ and some relative coordinates within each cell using a orthogonal coordinate transformation, the last 3 terms of $(13)$ can be decomposed into a c.m. Hamiltonian $H_{c . m}$. and a lattice sum of internal (relative coordinate) Hamiltonians $H_{r e l}=\sum_{n} H_{r e l, n}$, where $H_{r e l, n}$ contains only the $(N-1$ ) relative coordinates in cell $n$. Thus, only $H_{c . m}$. shows a coupling between the cells. This fact is completely analogous to the treatment of interacting quantum dot lattices considered in Ref. 16. Consequently, the total Hamiltonian (13) decomposes into 3 contributions

$$
H=E_{N}^{M a d}+H_{c m}+H_{r e l}
$$

which are considered in turn.

\section{A. Madelung energy}

Generally, we obtain $E_{N}^{M a d}$ from the conventional Madelung energy $(N=1)$ with the same lattice constant by $E_{N}^{M a d}=N^{2} E_{N=1}^{M a d}$. Here, however, we compare phases with the same mean density. In the hexagonal lattice, the Madelung energy per electron $\varepsilon_{N}^{M a d}$ for a given density parameter $r_{s}=1 / \sqrt{\pi n_{0}}$ can be deduced from the data in Ref. ๑ providing $\varepsilon_{N}^{M a d}=-1.106103 \beta N^{1 / 2} r_{s}^{-1}$.

\section{B. Center-of-mass energy}

The c.m. part of the Hamiltonian reads

$$
\begin{aligned}
H_{c m}= & \frac{1}{N}\left\{\sum_{n} \frac{1}{2 m^{*}}\left[\mathbf{P}_{n}+\frac{N}{c} \mathbf{A}\left(\mathbf{U}_{n}\right)\right]^{2}\right. \\
& \left.+\frac{N^{2}}{2} \sum_{n, n^{\prime}} \mathbf{U}_{n} \cdot \mathbf{C}_{n, n^{\prime}} \cdot \mathbf{U}_{n^{\prime}}\right\}
\end{aligned}
$$

with

$$
\begin{aligned}
\mathbf{C}_{n, n^{\prime}} & =+\beta N^{2} \mathbf{T}_{0} \quad \text { for } n=n^{\prime} \\
& =-\beta N \mathbf{T}_{n n^{\prime}} \quad \text { else }
\end{aligned}
$$

After the usual phonon transformation

$$
\begin{aligned}
& \mathbf{U}_{n}=\frac{1}{\sqrt{N_{c}}} \sum_{\mathbf{q}}^{B Z} e^{-i \mathbf{q} \cdot R_{n}^{0}} \mathbf{U}_{\mathbf{q}} \\
& \mathbf{P}_{n}=\frac{1}{\sqrt{N_{c}}} \sum_{\mathbf{q}}^{B Z} e^{+i \mathbf{q} \cdot R_{n}^{0}} \mathbf{P}_{\mathbf{q}}
\end{aligned}
$$

where $N_{c}$ is the number of cells, the Hamiltonian decouples with respect to the lattice sums and we obtain

$$
H_{c m}=\sum_{\mathbf{q}}^{B Z} H_{\mathbf{q}}
$$

with the magneto-phonon Hamiltonian 


$$
\begin{aligned}
H_{\mathbf{q}}= & \frac{1}{N}\left\{\frac{1}{2 m^{*}}\left[\mathbf{P}_{\mathbf{q}}+\frac{N}{c} \mathbf{A}\left(\mathbf{U}_{\mathbf{q}}^{*}\right)\right]^{\dagger} \cdot\left[\mathbf{P}_{\mathbf{q}}+\frac{N}{c} \mathbf{A}\left(\mathbf{U}_{\mathbf{q}}^{*}\right)\right]\right. \\
& \left.+\frac{N^{2}}{2} \mathbf{U}_{\mathbf{q}}^{*} \cdot \mathbf{C}_{\mathbf{q}} \cdot \mathbf{U}_{\mathbf{q}}\right\}
\end{aligned}
$$

and the dynamical matrix

$$
\mathbf{C}_{\mathbf{q}}=\sum_{n} e^{i \mathbf{q} \cdot \mathbf{R}_{n}^{0}} \mathbf{C}_{n, 0}=\beta N \sum_{n \neq 0}\left(1-e^{i \mathbf{q} \cdot \mathbf{R}_{n}^{0}}\right) \mathbf{T}_{n 0}
$$

The eigenvalues of $H_{c m}$ and $H_{\mathbf{q}}$ depend on $N$ only through the $N$ dependence of $\mathbf{C}$. The explicit $N$ dependence in (16) and (22) cancels. The last statement is obvious if we consider that the explicit $N$ can be removed by rescaling the displacement vector $\mathbf{U}$ by a factor of $\sqrt{N}$.

The eigenvalues of (22) are e.g. given in Ref. 16 .

$$
E\left(n_{+}, n_{-}\right)=\left(n_{+}+\frac{1}{2}\right) \omega_{+}+\left(n_{-}+\frac{1}{2}\right) \omega_{-} ; \quad n_{ \pm}=0,1,2, \ldots
$$

This provides a ground state energy

$$
E_{c m}=\sum_{\mathbf{q}}^{B Z} \frac{1}{2}\left(\omega_{+}+\omega_{-}\right)
$$

where

$$
\omega_{ \pm}=\sqrt{\frac{\omega_{c}^{* 2}}{2}+\tilde{\omega}_{0}^{2} \pm \sqrt{\frac{\omega_{c}^{* 4}}{4}+\omega_{c}^{* 2} \tilde{\omega}_{0}^{2}+\frac{\Delta^{2}}{4}+C_{12}^{2}}}
$$

with $\tilde{\omega}_{0}^{2}=\frac{1}{2}\left(C_{11}+C_{22}\right), \Delta=C_{11}-C_{22}, \omega_{c}^{*}=\frac{B}{m^{*} c}$ is the cyclotron frequency with the effective mass, and $C_{i, k}$ are the Cartesian components of $\mathbf{C}_{\mathbf{q}}$. If we define an auxiliary tensor $\mathbf{S}$ through $\mathbf{C}_{\mathbf{q}}=p \mathbf{S}_{\mathbf{q}}$ with $p=2 \beta N / a^{3}$ ( $a$ is the lattice constant), then $\mathbf{S}_{\mathbf{q}}$ depends only from the geometry of the lattice, i.e. it is a fixed tensor for the hexagonal and cubic lattice and depends only from the $b / a$ ratio for a rectangular lattice. The explicit form of $\mathbf{S}_{\mathbf{q}}$ is complicated and not of common interest. The sums involved have to be done numerically anyway. The interaction parameter $p$ for the hexagonal lattice reads in terms of the more convenient parameters $r_{s}$ and $n_{0}$

$$
p=2\left(\frac{\sqrt{3}}{2 \pi}\right)^{3 / 2} \beta r_{s}^{-3} N^{-1 / 2}=2\left(\frac{\sqrt{3}}{2}\right)^{3 / 2} \beta n_{0}^{3 / 2} N^{-1 / 2}
$$

Because of the optical selection rules, $\omega_{ \pm}$for $\mathbf{q}=0$ agree with the excitation energies $\Delta E$ for long wavelength (infrared) radiation.

It can be easily seen that for $B=0, \omega_{ \pm}=\sqrt{\text { eigenvalues }\left(\mathbf{C}_{\mathbf{q}}\right)}=\sqrt{p} \sqrt{\text { eigenvalues }\left(\mathbf{S}_{\mathbf{q}}\right)}$. Because of the universal character of $\mathbf{S}_{\mathbf{q}}$, the excitation energies in units of $\sqrt{p}$ comprise all data on the energies for hexagonal lattices in this limit (see Fig.1). In connection with formula (27) this means that the phonon frequencies of the paired WC, as compared with the single electron case of the same electron density, are smaller by a factor of $1 / \sqrt{2}$. Additionally, the frequencies (and the c.m. contribution to the total energy) for the same $N$ decay with the density parameter like $r_{s}^{-3 / 2}$. 


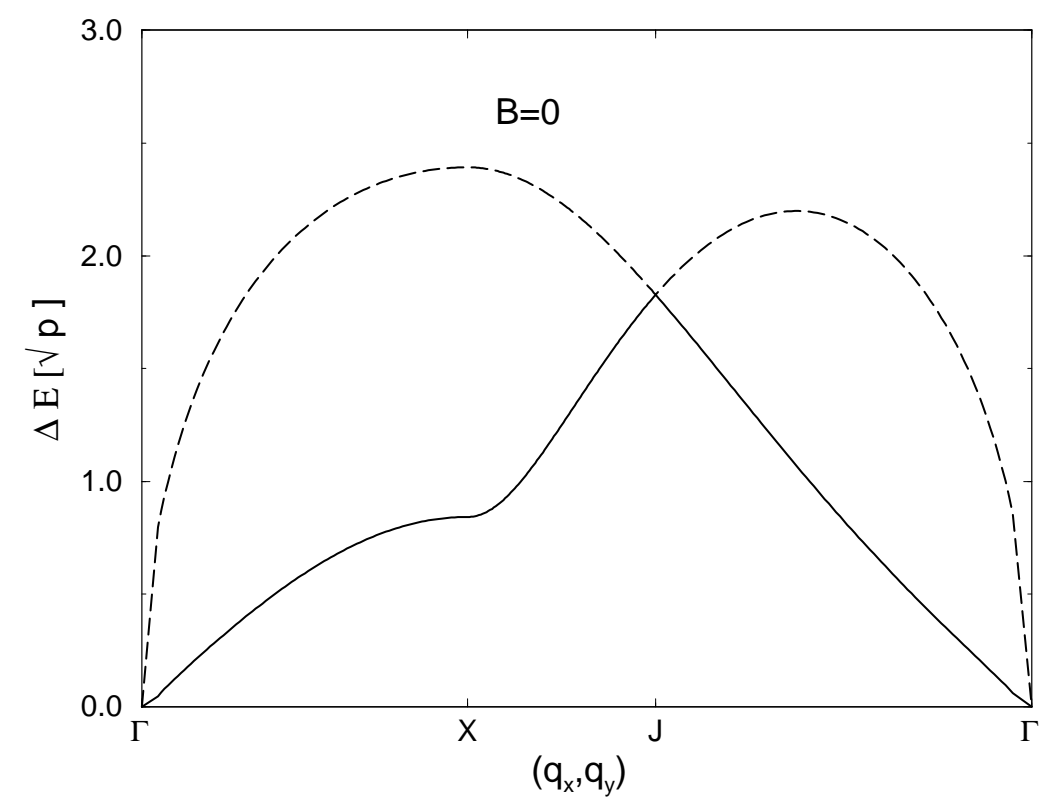

FIG. 1. Phonon dispersion (for $\mathrm{B}=0$ ) of the hexagonal Wigner lattice. (Energies are in units $\sqrt{p}$.)

For finite $B$, an universal function comprising all data cannot be defined. However, if we use $\omega_{c}^{*}$ as energy unit and $\omega_{c}^{* 2}$ as unit for the interaction parameter $p$, then the result depends only on $p$ and shows no explicit dependence on $B$.

For large $B$, (26) gives $\omega_{+}=\omega_{c}^{*}+\operatorname{trace}\left(\mathbf{C}_{\mathbf{q}}\right) / 2 \omega_{c}^{*}$ and $\omega_{-}=\operatorname{determinant}\left(\mathbf{C}_{\mathbf{q}}\right) / \omega_{c}^{*}$ (in agreement with Ref. B), and in the units mentioned above, this reads $\omega_{+}\left[\omega_{c}^{*}\right]=1+p\left[\left(\omega_{c}^{*}\right)^{2}\right] \cdot \operatorname{trace}\left(\mathbf{S}_{\mathbf{q}}\right) / 2$ and $\omega_{-}\left[\omega_{c}^{*}\right]=p\left[\left(\omega_{c}^{*}\right)^{2}\right] \cdot \operatorname{determinant}\left(\mathbf{S}_{\mathbf{q}}\right)$. Thus, the results are determined by the interaction parameter and two universal functions, namely $\operatorname{trace}(\mathbf{S})$ and determinant(S), which are shown in Fig.2. In the limit $B \rightarrow \infty$ we obtain the result of noninteracting electrons $\omega_{+}=\omega_{c}^{*}$ and $\omega_{-}=0$, as to be expected.

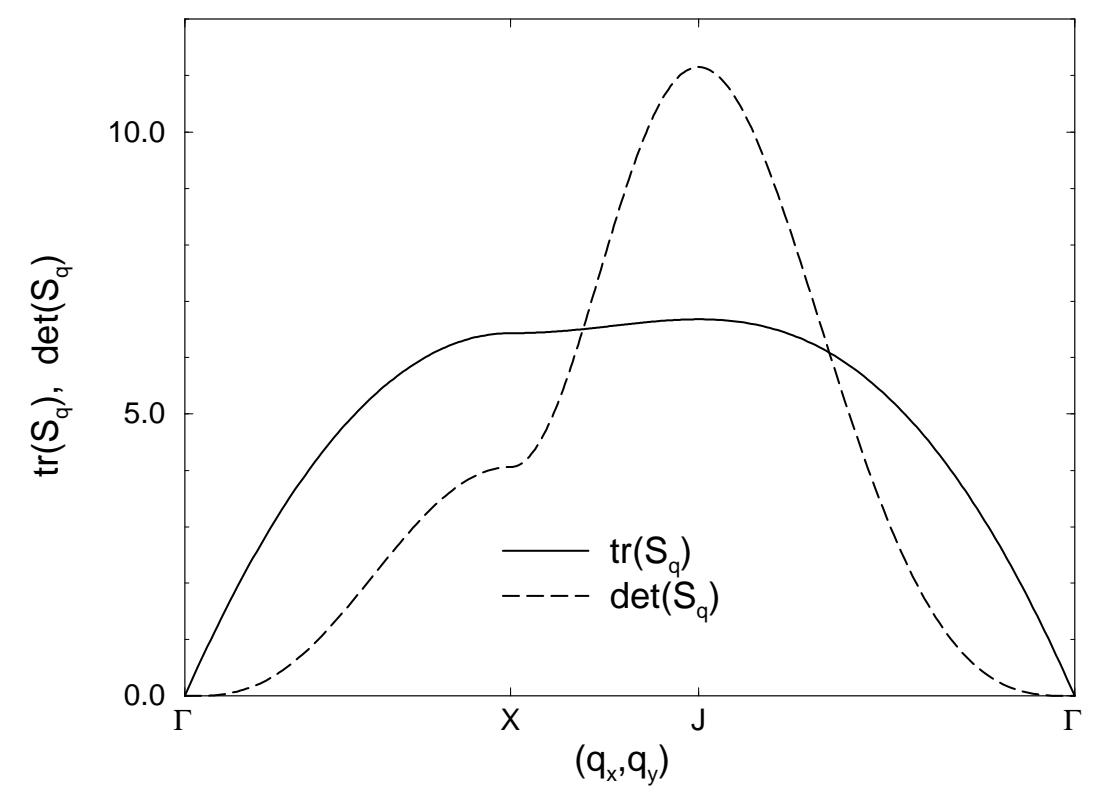

FIG. 2. The functions $\operatorname{trace}\left(\mathbf{S}_{\mathbf{q}}\right)$ and determinant $\left(\mathbf{S}_{\mathbf{q}}\right)$ determining the excitation frequencies in the high field limit. 
Direct comparison of excitation energies with experiments is not the aim of this work. What matters here is only the contribution of the 'zero point vibrations' to the total energy.

\section{Internal energy}

For two electrons per cell, the internal (relative coordinate) energy can be calculated easily. (Larger $\mathrm{N}$ call for a larger numerical effort and are postponed to a succeeding work.) If we introduce apart from the c.m. $\mathbf{U}_{n}$ the relative coordinate $\mathbf{u}_{n}$

$$
\begin{aligned}
\mathbf{U}_{n} & =\frac{1}{2}\left(\mathbf{u}_{n 1}+\mathbf{u}_{n 2}\right) \\
\mathbf{u}_{n} & =\mathbf{u}_{n 2}-\mathbf{u}_{n 1}
\end{aligned}
$$

then all relative Hamiltonians read (the index ' $n$ ' is omitted)

$$
H_{r e l}=2\left\{\frac{1}{2 m^{*}}\left[\mathbf{p}+\frac{1}{2 c} \mathbf{A}(\mathbf{u})\right]^{2}+\frac{1}{2} \mathbf{u} \cdot \mathbf{D} \cdot \mathbf{u}+\frac{\beta}{2 u}\right\}
$$

where $\mathbf{p}=-i \nabla_{\mathbf{u}}$ and $\mathbf{D}=\frac{\beta}{2} \mathbf{T}_{0}=\frac{1}{4} p S_{0} \mathbf{I}$ with $S_{0}$ being a lattice sum of the same type as the components of $S_{i k}$. (Do not mix up the interaction parameter $p$ and the vector of the momentum operator $\mathbf{p}$.) It should be emphasized that the electronic intercell interaction contributes a harmonic term to the intracell problem to $H_{r e l}$, namely the last but one in (refH-rel). As to the calculation of the eigenvalues of Hamiltonians of the form (30) we refer to Ref. 17. 


\section{RESULTS}

If not otherwise indicated, all results and parameters are given in effective atomic units for GaAs $\left(m^{*}=0.067, \beta=\right.$ $1 / 12$ ), .i.e., energies (and frequencies) in $1 a . u .^{*}=4.65 \cdot 10^{-4}$ doubleRydberg $=12.64 \mathrm{meV}$ and length in $1 a . u{ }^{*}=$ $1.791 \cdot 10^{2}$ Bohr $=0.9477 \cdot 10^{2} \stackrel{\circ}{A}$. (If the units of a quantity is indicated explicitly, then it is given in brackets after the quantity.) Observe that the effective density parameter in current typical experiments is of order 2.

\section{A. Contributions to the total energy}

For larger $r_{s}$, the energy difference between both phases is tiny (see Fig.3). As an example, at $r_{s}=10$ (at the right boundary of Fig.3) it amounts to $2 \cdot 10^{-4}$, calling for high precision computations (of order $10^{-5} a . u .^{*}$ ). This fact is amazing because the Madelung energy and the c.m. energy are both very different (see Fig.3). This difference is compensated almost completely by the relative energy of the paired phase.

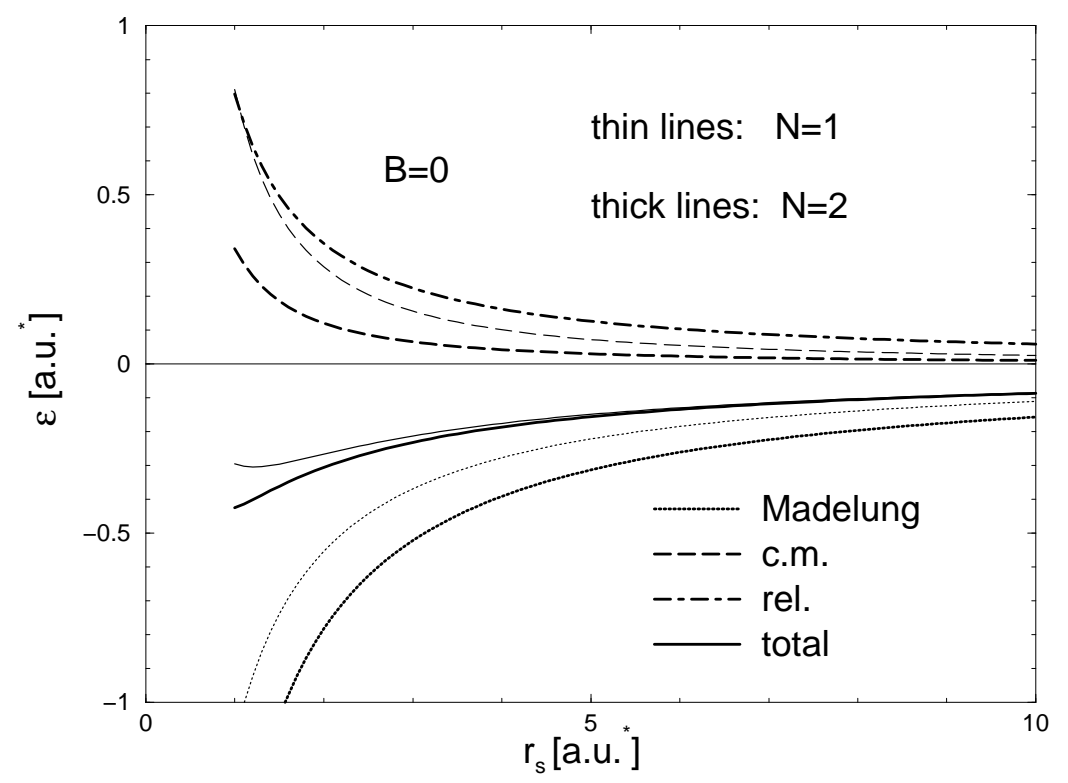

FIG. 3. Comparison of the contributions to the total energy in the single electron $(\mathrm{N}=1)$ and the paired (N=2) Wigner crystal for zero magnetic field.

\section{B. Phase diagram for single electron versus paired Wigner crystals}

Fig.4 shows the phase diagram for the single electron versus the paired WC phase in the $r_{s}-B$ plane. In shaded regions, the electron pairs are in singlet state and in triplet states elsewhere. The spin configuration of the paired phase has been determined irrespective of the question, if the single electron phase is below both paired (singlet or triplet) phases. The thin full lines separate regions with different relative (internal) angular momentum $m$ of the pairs, which has the lowest energy. The value of $m$ is indicated in each region as well. (Even $m$ belongs to the state and odd $m$ to the triplet state.) The thick full line separates the regions where the single electron WC is the ground state from regions, where the paired WC has the lower energy. We want to emphasize that this figure does not say anything about the issue if any other (e.g. liquid) phase is lower in energy than the phases considered here. The kinks in the phase boundary are connected with changes of the total spin of the paired phase. 

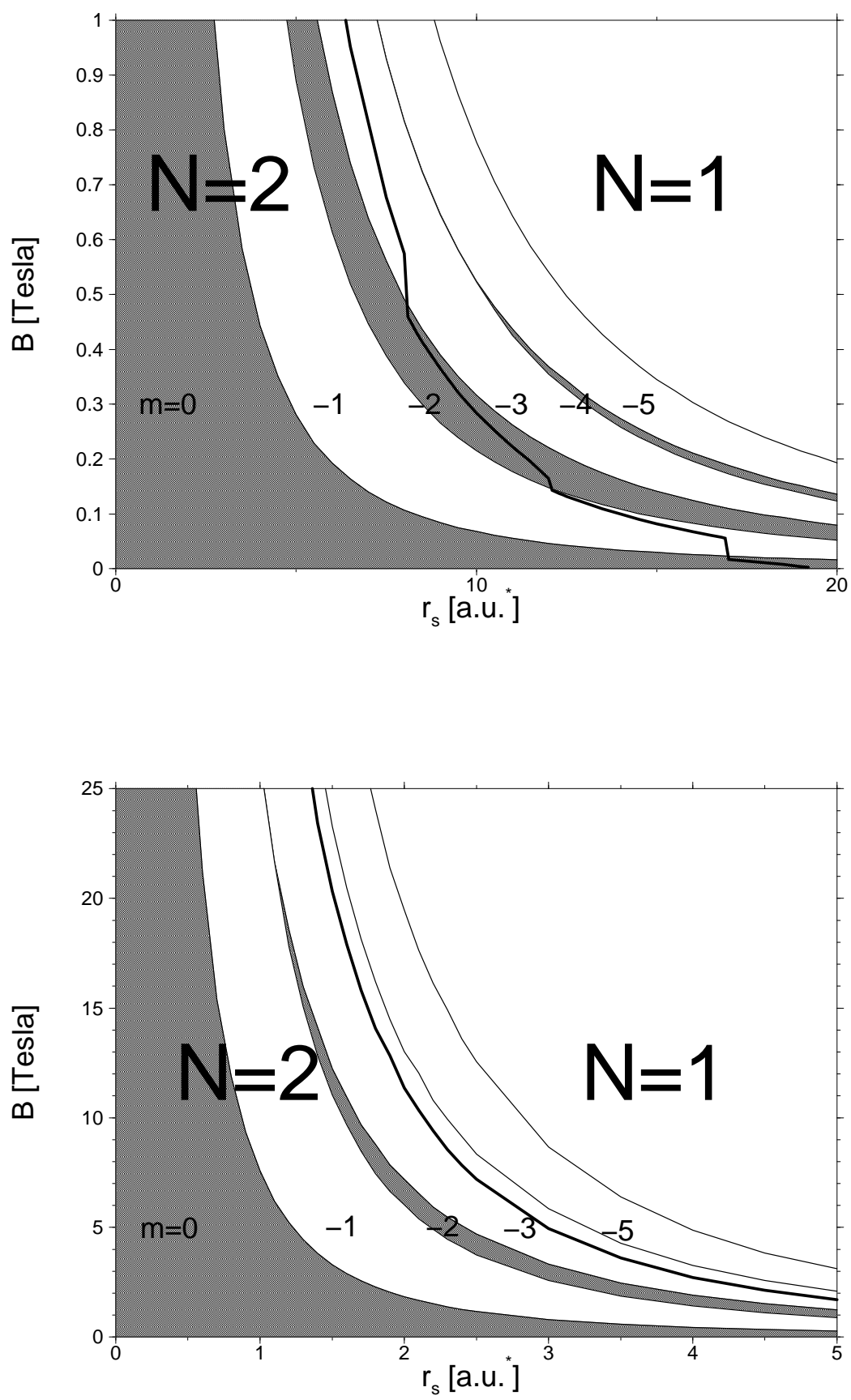

FIG. 4. Phase diagram in the $r_{s}-B$ plane (see text for details) a) for small $B$ and b) for small $r_{s}$.

First, we discuss the case $B=0$. For $r_{s}$ above 19, the energetically lower state is the single electron WC and below it is the paired phase. Clearly, in the limit of high densities (small $r_{s}$ ) any liquid state should win. It is physically imaginable, that in increasing the density, the state starts with the single electron WC, then adopts the paired state before it goes over to the liquid. If there are states with $3,4, \ldots$ electrons involved, will be the focus of further investigations.

For a certain finite $B$, the density, where the transition to the paired ground state occurs, becomes higher. In other 
words, for higher $B$ one needs less dilute electron systems to produce a single electron WC. This is physically obvious, because the magnetic field helps to localize the electrons. In other words, lowering the density cases pair breaking. Now we discuss the pair breaking process as a function of $B$ for fixed $r_{s}$, say $r_{s}=2$. If we start with $B=0$ and increase $B$, the paired state undergoes first some spin transitions from singlet to triplet and vice versa before the pairs break in the triplet state. It should be mentioned that pair breaking and spin transition are not coupled together. The reason is that the Zeeman energy is too small to play a decisive role.

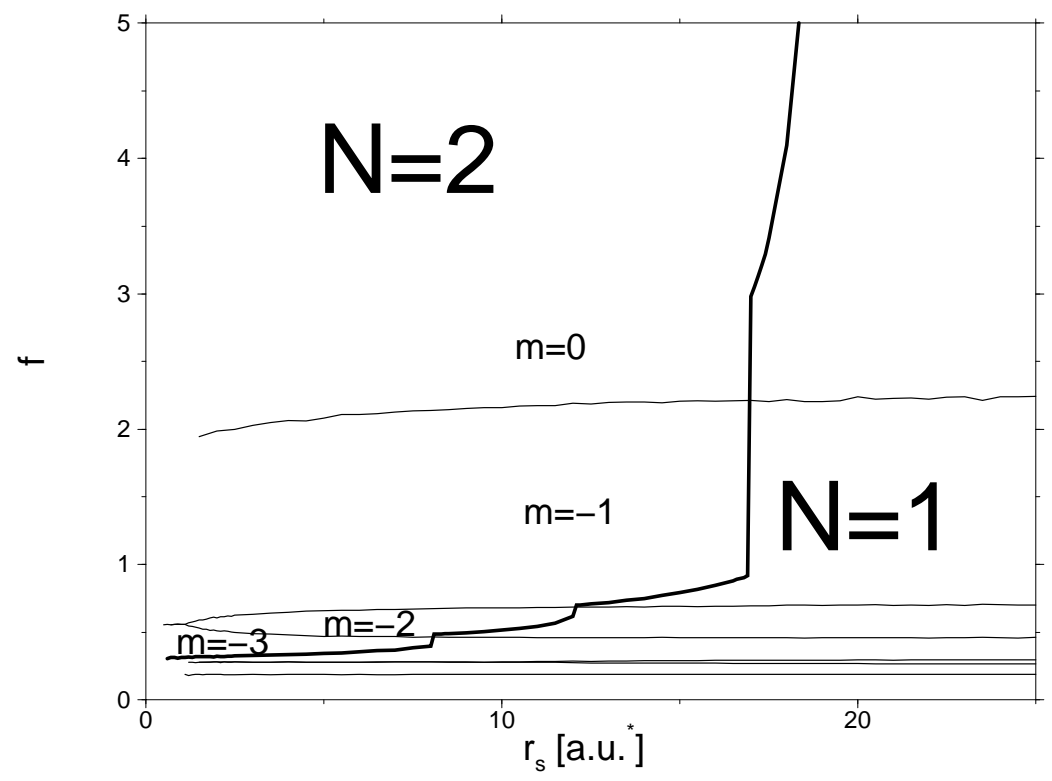

FIG. 5. Phase diagram in the $r_{s^{-}} f$ plane (see text for details).

Fig.5 shows the the phase diagram again, but the magnetic field axis is replaced by the filling factor $f=$ $14.64 /\left(B[T] r_{s}^{2}\left[a . u .^{*}\right]\right)$ (using the parameters of GaAs). This plot has the advantage of showing the physically important parameter $f$ directly, but does not allow the discussion of the limit $B=0$, because it is transformed to $f \rightarrow \infty$.) Again, thin lines separate regions with different $m$ (and therefore total spin) of the paired phase and the thick line separates the single electron WC ground state from the paired WC. As to be expected, the single electron WC is restricted to the low density - small filling factor region. It is clear that in a complete phase diagram, the region, which is here attributed to the paired phase, can be further restricted by the inclusion of all kinds of liquid phases (and possibly WC phases with $\mathrm{N}=2,3, \ldots$ electrons per cell).

\section{Comparison of Wigner crystal phases with Laughlin liquid}

Because the Laughlin liquid is restricted to discrete filling factors, we compare in Fig.6 the energy (or, more conveniently, $\left.r_{s}\left(\varepsilon-\omega_{c} / 2\right)\right)$ of different phases for fixed $f=1 / 3$ as a function of $r_{s}$. The Zeeman energy is omitted in this figure because the paired phase is triplet over the whole $r_{s}$ range. Therefore it would have contributed for a given $r_{s}$ the same amount to all phases, but introduce an unpleasant pole at $r_{s}=0$ into the curves. (In all calculations, however, the Zeeman energy has to be taken into account because of the different spin configurations in the paired WC.) The curve for the Laughlin liquid from Ref. 19 includes Landau level mixing (as our results do) by adding a variational correlation factor to the Laughlin function. (The value at $r_{s}=0$ corresponds to the result of the original Laughlin function.) As seen in Fig.6, the Laughlin liquid and the single electron WC are the ground state for $r_{s}$ below and above 15, respectively. This is in qualitative agreement with experiment. In our result for $f=1 / 5$ (not shown), the single electron $\mathrm{WC}$ is the ground state over the whole range of densities. This is not quite correct, because experiments suggest that the domain of the $\mathrm{WC}$ begins at $f=1 / 7$. Inclusion of a variatignal correlation factor to the WC wave-function fixes the phase boundary between the Laughlin liquid and the WC20 21 . This amendment could not be added here, because it would have become too complicated for the paired WC and in comparing both WC phases we needed a description on the same footing. 


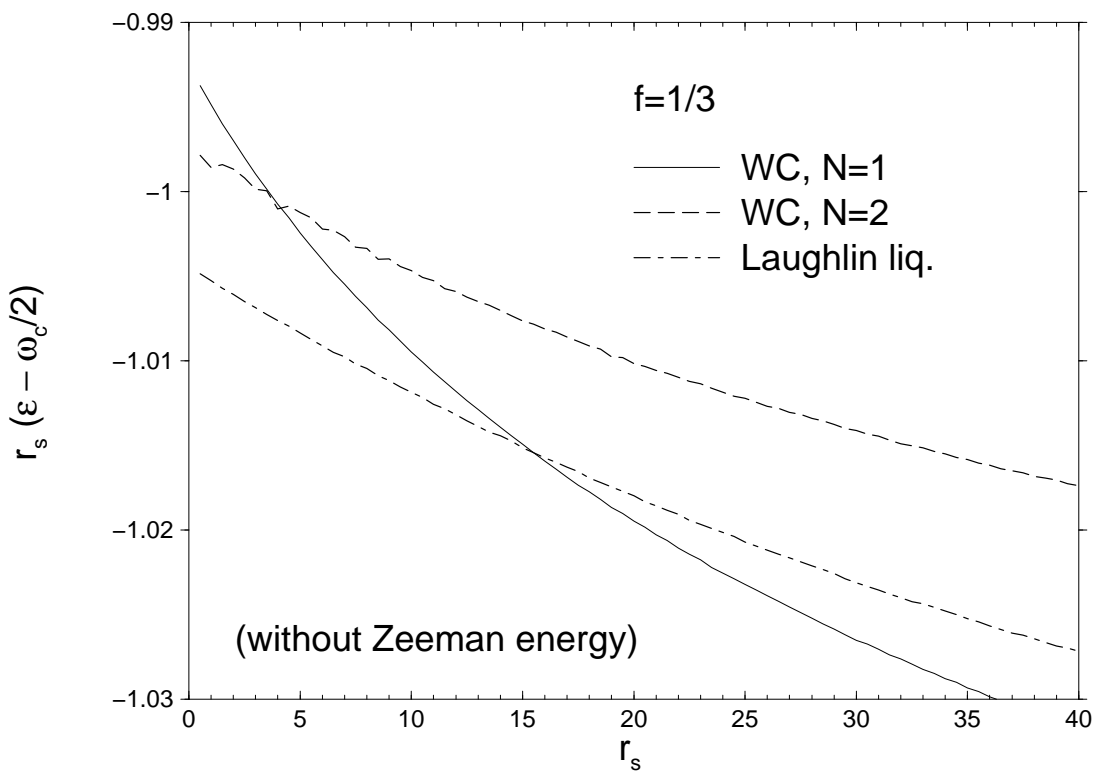

FIG. 6. Comparison of the Wigner crystal states with the Laughlin liquid.

\section{SUMMARY AND OVERVIEW}

We have shown that the paired WC is energetically more favorable than the single electron WC for higher densities and lower magnetic fields. If we start with the paired WC, lowering the density and increasing the magnetic field will break the pairs. At least in GaAs, the Zeeman energy plays a less important role than the angular momentum dependence of the internal (relative coordinate) energy. Observe that changing the internal angular momentum (from even to odd) is coupled with a change of the total spin of each cluster due to the Pauli principle. Therefore, the paired phase undergoes first some singlet- triplet transitions before the pairs break and the single electron WC is reached. The singlet- triplet transitions have the same physical origin as the corresponding transitions in quantum dots, because they are driven by the internal energy, which is similar in either system.

In our approach, the interaction within each cluster is exactly accounted for (including exchange and correlations). In both cases, the intercell interaction is considered in second order van der Waals approximation (no exchange, Coulomb interaction in dipole approximation). The validity of the second order approximation has been checked in Ref. 18 by estimating the third order contribution, which amounts to a few \%. We did not include higher order contributions from two reasons. First, they spoil the exact decoupling and make the paired WC intractable. Second, these corrections have most likely the same sign in the single electron and the paired phase and they cancel partly in comparison of both energies. The neglect of inter-cell exchange (but keeping the Coulomb correlations) is justified in the low density and high magnetic field limit, because the exchange energy drops off exponentially, but the correlation energy like $1 / r$. It seems to be worthwhile to elaborate on the differences of this work and Ref. 12. They targeted on high LLs (low magnetic fields), and high electron densities $\left(N_{L L}>>r_{s}^{-1}>>1\right)$. LL mixing and incomplete spin polarization are ruled out and the completely filled LLs are frozen out and influence the upper LL only through a screening in the $\mathrm{e}-\mathrm{e}$ - interaction. Therefore we called both treatments in the abstract 'complementary'. What seems to be interesting is that despite approaching reality with different assumptions and virtually from opposite sides, the effect of clusterization of the liquids is found in both treatments. 
${ }^{1}$ K.Maki and X.Zotos, Phys.Rev.B 28, 4349 (1983)

${ }^{2}$ D.Yoshioka and P.A.Lee, Phys.Rev.B 27, 4986 (1983); D. Yoshioka, Phys.Rev.B 29,6833 (1984); and Surface Sci. 170, 125 (1986)

${ }^{3}$ R.Cote and A.H.MacDonald, Phys.Rev.Lett. 65, 2662 (1990) and Phys.Rev.B 44, 8759 (1991)

${ }^{4}$ L.Bonsall and A.A.Maradudin, Phys. Rev.B 15, 1959 (1977)

${ }^{5}$ B.I.Halperin, Helvetica Physica Acta 56, 75 (1983)

${ }^{6}$ M.Greiter, Xiao-Gang Wen, and F.Wilczek, Phys. Rev. Lett. 66, 3205 (1991)

${ }^{7}$ N.Read, G.Moore, Progr. Theoret. Phys. Suppl. 107, 257 (1992)

${ }^{8}$ R.H.Morf, Phys. Rev. Lett. 80, 1505 (1998)

${ }^{9}$ F.D.M.Haldane, E.H.Rezayi, Phys. Rev. Lett. 60, 956 (1988) and E.H.Rezayi, F.D.M.Haldane, Phys. Rev. Lett. 84, 4685 (2000)

${ }^{10}$ K.Moulopoulos, N.W.Ashcroft, Phys.Rev.Lett. 69, 2555 (1992) erratum: 70, 2356 (1993)

${ }^{11}$ M.Taut, Solid State Commun.89, 189 (1994)

12 A.A.Koulakov, M.M.Fogler and B.I.Sklovskii, Phys.Rev.Lett. 76, 499 (1996) and Phys.Rev. B 54, 1853 (1996)

${ }^{13}$ F.D.M.Haldane, E.H.Rezayi, and Kun Yang, Phys.Rev.Lett. 85, 5396 (2000), and ibid. 83, 1219 (1999)

${ }^{14}$ N.Shibata, D.Yoshioka, preprint cond-mat 0101401

${ }^{15}$ M.M.Fogler and A.A.Koulakov, Phys.Rev.B 55, 9326 (1997)

${ }^{16}$ M.Taut, cond-mat 0002155 and Phys.Rev.B 62, 8126 (2000) In the latter, at the r.h.s. of formula (16) after the $\prod$ symbol, the symbols $Q=$ have to be ommitted.

${ }^{17}$ M.Taut, J.Phys.A27, 1045 (1994) miss-prints corrected in erratum J.Phys.A27, 4723 (1994). Additionally, in formula (10) in the term containing $\frac{\partial}{\partial \alpha}$ a factor $\frac{1}{2}$ is missing, and on the r.h.s. of (19a) and (20a) $\tilde{\omega}$ must be replaced by $\tilde{\omega}_{r}$.

${ }^{18}$ K.Esfarjani and S.T.Chui, Phys.Rev.42, 10758 (1990); and J.Phys.Condens.Matter 3, 5625 (1991)

${ }^{19}$ R.Price, P.M.Platzman, and S.He, Phys.Rev.Lett. 70, 339 (1993); P.M.Platzman and R.Price, Phys.Rev.Lett. 70, 3487 (1993)

${ }^{20}$ X.Zhu and S.G.Louie, Phys.Rev.Lett. 70, 335 (1993); and Phys. Rev. B 52, 5863 (1995)

${ }^{21}$ P.K.Lam and S.M.Girvin, Phys.Rev.B 30, 473 (1984) 